\title{
GIP increases adipose tissue expression and blood levels of MCP-1 in humans and links high energy diets to inflammation: a randomised trial
}

\author{
Özlem Gögebakan ${ }^{1,2} \cdot$ Martin A. Osterhoff ${ }^{1,2} \cdot$ Rita Schüler $^{1} \cdot$ Olga Pivovarova $^{1,2}$. \\ Michael Kruse $^{1}$ - Anne-Cathrin Seltmann ${ }^{1}$ • Alexander S. Mosig ${ }^{3} \cdot$ Natalia Rudovich $^{1,2}$ • \\ Michael Nauck ${ }^{4}$ - Andreas F. H. Pfeiffer ${ }^{1,2}$
}

Received: 19 February 2015 / Accepted: 15 April 2015 /Published online: 21 May 2015

(C) Springer-Verlag Berlin Heidelberg 2015

\begin{abstract}
Aims/hypothesis Obesity is associated with elevated monocyte chemoattractant protein-1 (MCP-1), a proinflammatory chemokine related to diabetes and cardiovascular disease. Since obesity is triggered by energy dense diets, we hypothesised that nutrient induced intestinal hormones such as glucose-dependent insulinotropic peptide (GIP) may directly stimulate the release of chemokines from adipose tissue and induce low-grade inflammation.

Methods GIP effects on gene expression and secretion of inflammatory markers were studied by microarray analysis and PCR from human subcutaneous fat biopsies of slightly obese but healthy volunteers in the metabolic ward of German Institute of Human Nutrition, Department of Clinical Nutrition, Potsdam-Rehbrücke. To allocate the participants
\end{abstract}

Özlem Gögebakan and Martin A. Osterhoff contributed equally to this study.

Electronic supplementary material The online version of this article (doi:10.1007/s00125-015-3618-4) contains peer-reviewed but unedited supplementary material, which is available to authorised users.

Martin A. Osterhoff

martino@dife.de

1 Department of Clinical Nutrition, German Institute of Human Nutrition, Potsdam-Rehbruecke, Arthur-Scheunert-Allee 155, 14558 Nuthetal, Germany

2 Department of Endocrinology, Diabetes and Nutrition, Charité University Medicine Berlin, Campus Benjamin Franklin, Berlin, Germany

3 Research Group Molecular Hemostaseology, Jena University Hospital, Jena, Germany

4 Diabetes Zentrum Bad Lauterberg, Bad Lauterberg, Germany to the study arms they were numbered in order of their recruitment and then assigned to the groups by a random number generator. In a randomised, single-blind (participants) crossover design, the participants received GIP infusions in postprandial concentrations $\left(2 \mathrm{pmol} \mathrm{kg}{ }^{-1} \mathrm{~min}^{-1}\right)$ or saline (154 mmol/1 NaCl) infusions for $240 \mathrm{~min}$ either alone, in combination with hyperinsulinaemic-euglycaemic (EU) or hyperinsulinaemic-hyperglycaemic (HC) clamps. Possible mechanisms of GIP effects were investigated in single and co-cultures of macrophage and adipocyte cell lines and in primary human monocytes, macrophages and adipocytes.

Results A total of 17 participants were randomised to the following groups: EU with GIP infusion $(n=9) \mathrm{EU}$ with $\mathrm{NaCl}$ infusion $(n=9) \mathrm{HC}$ with GIP infusion $(n=8)$; $\mathrm{HC}$ with $\mathrm{NaCl}$ infusion $(n=8)$ sole GIP infusion $(n=11)$ and sole placebo infusion $(n=11)$. All 17 individuals were analysed. The study is completed. In human subcutaneous adipose tissue (hSCAT), infusions of GIP significantly increased inflammatory chemokine and cytokine gene networks in transcriptomic microarray

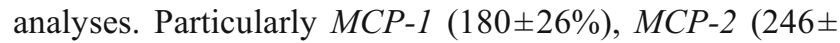
$58 \%)$ and $I L-6(234 \pm 40 \%)$ mRNA levels in adipose tissue as well as circulating plasma concentrations of MCP-1 $(165 \pm 12$ vs $135 \pm 13 \mathrm{pg} / \mathrm{ml}$; GIP vs saline after $240 \mathrm{~min}$; $p<0.05$ for all variables) in humans increased independently of circulating insulin or glucose plasma concentrations. GIP stimulation increased Mcp-1 mRNA-expression in co-cultures of differentiated 3T3L1-adipocytes and RAW 264.7 macrophages but not in the isolated cell lines. Similarly, GIP increased $M C P-1$ transcripts in co-cultures of primary human macrophages with human adipocytes. GIP receptor $(G I P R)$ transcripts were present in primary monocytes and the different cell lines and induced activation of extracellular related kinase (ERK) as well as increases in cAMP, indicating functional receptors. 
Conclusions/interpretation Our findings suggest that the nutrient induced gut hormone GIP may initiate adipose tissue inflammation by triggering a crosstalk of adipocytes and macrophages involving MCP-1.

Trial registration: ClinicalTrials.gov NCT00774488

Funding: This work was supported by the German Research Foundation (DFG): grant No. Pf164/021002

Keywords Adipose tissue - GIP - Inflammation - MCP-1

$\begin{array}{ll}\text { Abbreviations } \\ \text { CCR } & \text { C-C chemokine receptor } \\ \text { ERK } & \text { Extracellular related kinase } \\ \text { EU } & \text { Euglycaemic } \\ \text { GIP } & \text { Glucose-dependent insulinotropic peptide } \\ \text { GIPR } & \text { GIP receptor } \\ \text { HC } & \text { Hyperglycaemic } \\ \text { hSCAT } & \text { Human subcutaneous adipose tissue } \\ \text { iGA } & \text { Iterative group analysis } \\ \text { MAPK } & \text { Mitogen activated protein kinase } \\ \text { MCP } & \text { Monocyte chemoattractant protein } \\ \text { MIP-1 } \beta & \text { Macrophage inflammatory protein-1 } \beta \\ \text { PBMC } & \text { Peripheral blood mononuclear cell } \\ \text { PCA } & \text { Principal component analysis } \\ \text { qPCR } & \text { Quantitative PCR } \\ \text { SGBS } & \text { Simpson-Golabi-Behmel syndrome } \\ \text { WGCNA } & \text { Weighted gene co-expression network analysis }\end{array}$

\section{Introduction}

Obesity and insulin resistance are associated with increased infiltration of adipose tissue with classically activated (M1) macrophages, which are a substantial source of multiple cytokines [1,2]. These local inflammatory factors, such as TNF- $\alpha$ and IL- 6 are thought to act directly on adipocytes to affect their insulin sensitivity and biological responses [3-5]. The crosstalk between adipocytes and macrophages was shown to be a crucial mechanism aggravating inflammatory changes in adipose tissue [6]. Experimental inhibition of the macrophage inflammatory responses or phenotype was shown to markedly improve insulin sensitivity $[7,8]$.

While a role for chemokines in macrophage recruitment is well established, the initiation of these signals is not fully understood. One of the most important candidates is monocyte chemoattractant protein-1 (MCP-1), which is highly expressed in adipose tissue by both adipocytes and macrophages, and potently recruits monocytes/macrophages into adipose tissue $[5,9,10]$. Moreover, MCP-1 was recently shown to regulate adipocyte biology directly by altering their differentiation and insulin sensitivity [11]. Transgenic mice that overexpress $M c p-1$ specifically in adipocytes develop adipose tissue inflammation and insulin resistance without obesity $[9,10]$.

Since obesity is triggered by diets high in fat and energy content, nutrient induced hormones such as glucosedependent insulinotropic peptide (GIP) may directly stimulate the release of chemokines from adipose tissue.

Both fat and glucose are potent stimulants of the neuroendocrine gut hormone GIP, an incretin synthesised by endocrine $\mathrm{K}$ cells in the upper intestine, which responds very rapidly to food intake and is well known for its insulinotropic actions [12-15]. Deficiency of the GIP receptor (GIPR) is associated with pronounced metabolic improvement in animal models of diet-induced obesity [16-18]. Additionally, obesity appears to coincide with elevated GIP plasma levels in humans [13, 19]. GIPRs are known to be present on adipocytes and were shown to play a role in the regulation of glucose and lipid metabolism as well as in adipocyte differentiation [20-23]. The expression level of GIPRs in humans was highly correlated with body weight, waist circumference, insulin levels and insulin sensitivity, as well as with triacylglycerols and HDL-cholesterol [24]. In recent cell culture experiments with human primary adipocytes, GIP was shown to elicit inflammatory responses involving IL-1 $\beta$ and IL-6 [25].

We therefore investigated in a clinical trial whether treatment of human participants with GIP acutely affects inflammatory responses, in particular the expression of $M C P-1$ in adipose tissue and its circulating levels with respect to a possible GIP-dependent recruitment of macrophages. Moreover, we investigated the effects of GIP on $M C P-1$ mRNAexpression in human adipose tissue, adipocytes and macrophages in vitro separately as well as in co-cultures. We further examined whether functional GIPRs were expressed on monocytes and macrophages.

\section{Methods}

For a more detailed description, please refer to the electronic supplementary material (ESM) Methods.

Participants The study was approved by the ethics commission of Brandenburg, Germany (Registration No. AS 2(a)/ 2005). All individuals gave written informed consent prior to the study.

We studied 17 healthy male obese (BMI $>28$ ) participants with normal glucose tolerance in the range of 18 to 70 years of age whose characteristics are summarised in ESM Table 1. All participants had no evidence for metabolic disease and reported a stable body weight for at least 12 months. They were instructed to avoid significant physical activity 3 days preceding each examination day and to use a food intake protocol throughout the study period. 
Study design All studies and examinations were done in the overnight $(>10 \mathrm{~h})$ fasted state. Each participant underwent a complete physical examination and evaluation of medical history, including standard fasting laboratory and clinical chemistry as well as OGTT with $75 \mathrm{~g}$ glucose (Roche Diagnostics, Mannheim, Germany) to assess the metabolic state.

The effect of synthetic human GIP (1-42) (PolyPeptide Laboratories, Wolfenbüttel, Germany) administration on gene expression in human subcutaneous adipose tissue (hSCAT) was studied under three different conditions in a single blind design (ESM Fig. 1). Either the participants received only a GIP $\left(2 \mathrm{pmol} \mathrm{kg}{ }^{-1} \mathrm{~min}^{-1}\right)$ or a saline $(154 \mathrm{mmol} / \mathrm{l} \mathrm{NaCl})$ infusion for $240 \mathrm{~min}$. At different investigation days participants underwent hyperinsulinaemic-euglycaemic (EU, capillary glucose concentration $4.44 \mathrm{mmol} / \mathrm{l})$ and -hyperglycaemic ( $\mathrm{HC}$, $7.8 \mathrm{mmol} / \mathrm{l}$ ) clamps (insulin infusion rate: $40 \mathrm{mU} \mathrm{m}^{-2} \mathrm{~min}^{-1}$ ) combined with GIP or saline infusions $(0.9 \% \mathrm{NaCl}$ isotonic solution, Fresenius, Bad Homburg, Germany) for $240 \mathrm{~min}$ in a randomised, single-blind, crossover design. From each participant, two hSCAT biopsies were taken before and after infusions. Between intervention days, an intermission time of at least 7 days was maintained.

\section{Insulin, GIP and MCP-1 ELISA in human blood} samples Insulin was determined in serum by insulin-ELISA (Mercodia, Uppsala, Sweden). In plasma containing aprotinin (Trasylol, Bayer, Leverkusen, Germany), human GIP (total) ELISA kit (Linco Research, St Charles, MO, USA) was used and MCP-1 in plasma measured by human MCP-1 Quantikine kit (R\&D Systems, Wiesbaden, Germany).

Analysis of hSCAT biopsies Frozen hSCAT $(300 \mathrm{mg}$ ) was extracted with QIAzol and RNA extracted with Lipid Tissue RNA-Kit (Qiagen, Hilden, Germany). Total RNAs were processed with Agilent One Color Spike Mix Stock Solution and hybridised to a total number of 100 Agilent 60-mer Whole human Genome $(4 \times 44 \mathrm{~K})$ single-colour DNA microarrays (Agilent Technologies, Waldbronn, Germany). Expression microarray data was uploaded to NCBI GEO (www.ncbi. nlm.nih.gov/geo/query/acc.cgi?acc=GSE40589); Accession No: GSE40589.

In addition, cDNA was generated with the high-Capacity cDNA Reverse Transkription Kit (Applied Biosystems, Darmstadt, Germany). Each cDNA was labelled by Power SYBR Green master mix and detected with the ABI Prism 7900HT Sequence Detector in triplicates by use of the standard curve method (Applied Biosystems, Darmstadt, Germany). Samples were normalised to ribosomal protein large protein 0 (RPLP0) and fold changes calculated.

Culture of single cell-lines, co-culture, and media-transfer of adipocytes and macrophages THP-1, RAW 264.7,
3T3-L1 and Simpson-Golabi-Behmel syndrome (SGBS) cells were maintained at $37^{\circ} \mathrm{C}$ and $5 \% \mathrm{CO}_{2}$.

Human primary blood monocytes were isolated from whole blood samples using RosetteSep Monocyte Enrichment Kit (STEMCELL Technologies, Vancouver, Canada). Peripheral blood mononuclear cell (PBMC) fractions were extracted by gradient centrifugation with Ficoll N (GE-Healthcare, München, Germany). Monocytes were isolated to high purity ( $\geq 95 \%$ ) by anti-CD14 magnetic cell sorting (Miltenyi Biotec, Bergisch Gladbach, Germany). Human primary monocytes were differentiated to macrophages with granulocyte macrophage colony-stimulating factor (GM-CSF; PeproTech, Hamburg, Germany).

For co-culture, either RAW 264.7 macrophages $\left(2 \times 10^{5}\right.$ cells) were plated onto differentiated 3T3-L1-adipocytes $\left(\approx 2.5 \times 10^{6}\right)$ or human monocyte-derived macrophages $(\approx 1 \times$ $10^{5}$ cells) were plated onto differentiated SGBS cells $(\approx 3.5 \times$ $10^{5}$ per well) for $240 \mathrm{~min}$ in contact with each other under serum-starved conditions before GIP stimulation. As a control, adipocytes and macrophages were cultured separately but in equal numbers as in co-cultures and mixed after harvest.

For the study of gene expression, $100 \mathrm{nmol} / \mathrm{l} \mathrm{GIP}$ was added for $240 \mathrm{~min}$ or $24 \mathrm{~h}$. To avoid degradation of GIP, $25 \mu \mathrm{mol} / 1$ diprotin A was added, or GIP analogue GIP-D2Ala was used.

To determine whether adipocytes or macrophages were mainly responsible for the cytokine production in co-cultures of both cell lines, media-transfer experiments were carried out.

Cyclic adenosine monophosphate measurement Human primary blood monocytes, THP-1 and RAW 264.7 cells were plated at a concentration of $3 \times 10^{5}$ per $50 \mu \mathrm{l}$ per well in serum free medium $24 \mathrm{~h}$ prior to stimulation. Cells were incubated with $500 \mathrm{nmol} / 1$ 3-isobutyl-1-methylxanthine (IBMX) and either $\mathrm{NaCl}(0.9 \%)$ or GIP $100 \mathrm{nmol} / \mathrm{l}$ for different times $(2-30 \mathrm{~min})$. cAMP concentrations were determined with the cAMP-Screen Direct System (Applied Biosystems, Bedford, MA, USA).

p44/42-mitogen activated protein kinase activation assay Cells were plated $\left(5 \times 10^{6} / 10 \mathrm{ml}\right)$ and serum starved for $24 \mathrm{~h}$ prior to stimulation. Cells were incubated with GIP $100 \mathrm{nmol} / 1$ or $\mathrm{NaCl}$ solution $(0.9 \%)$ containing diprotin $\mathrm{A}$, for $30 \mathrm{~min}$ and subsequently harvested.

Western blotting Whole protein lysates were incubated with either GIPR antibodies (1:100), anti-p44/42 mitogen activated protein kinase (MAPK) or anti-phospho-p44/42 MAPK antibodies $(1: 2,000)$, followed by detection with a goat anti-rabbit IgG-HRP linked antibody $(1: 1,000)$ or horse anti-mouse IgG-HRP linked antibody $(1: 1,000)$ and the chemoluminescence system LumiGLO (Cell Signaling Technology, Beverly, MA, USA). 
Statistical analysis All data are given as means \pm SEM. Statistical analysis was performed using SPSS 14.0 (SPSS, Chicago, IL, USA). To test for normal distribution the Kolmogorov-Smirnov test was used. A paired, two-tailed Student's $t$ test was used to test for statistical significance between groups. A $p$ value $<0.05$ was assumed as significant.

The evaluation of microarrays was performed using Agilent GeneSpring GX Version 11 Software (Agilent Technologies, Waldbronn, Germany). Statistical significance of expression changes was calculated by unpaired $t$ test for two groups, $p$ value calculation was asymptotic and corrected for multiple testing by the Benjamini-Hochberg method. Iterative group analysis (iGA) [26] and weighted gene coexpression network analysis (WGCNA) [27] were used for analysis of regulated gene-clusters.

Real-time quantitative PCR (qPCR) variables and plasma MCP-1 concentrations were evaluated by ANOVA with intervention setup (infusion, EU-, HC-clamp) and treatment (GIP infusion or saline infusion) as factors. The model was adjusted for age, WHR, plasma glucose and plasma insulin concentration.

\section{Results}

We wanted to investigate the effects of either GIP alone or in combination with insulin and glucose in hSCAT of metabolically healthy but moderately overweight volunteers (ESM Table 1). We therefore performed infusions of GIP either alone or under hyperinsulinaemic, EU and HC conditions in a placebo $(0.9 \%$ saline solution) controlled manner for $240 \mathrm{~min}$. The HC condition was chosen to mimic the postprandial state, showing the combined action of GIP, glucose and insulin, while the EU condition would allow for investigating interactions of GIP and insulin in postprandial concentrations, independent of elevated glucose levels. GIP infusion led to GIP plasma concentrations of $\sim 120 \mathrm{pmol} / 1$ compared with $\sim 5 \mathrm{pmol} / 1$ during saline infusion (ESM Table 2, $p<0.001$ ), which resembled the postprandial and fasted states, respectively. Plasma insulin, capillary glucose and plasma GIP concentrations during the clamps and infusions are shown in ESM Table 2.

\section{Regulation of inflammatory gene networks and single} genes in microarray data We first analysed the microarray dataset by iGA [26], to find known GIP-regulated pathways, and WGCNA [27], to find unknown GIP-regulated networks by detecting gene sets with high inter-connectivity.

WGCNA WGCNA clustered 10,000 pre-selected genes into up to 15 modules depending on the experimental treatment revealing a variety of genes that changed the module they belonged to treatment dependently. Interestingly, when subjecting the distribution to gene modules to a principal component analysis (PCA), exactly two components were extracted referring either to all saline infusions or all experiments with GIP infusion, demonstrating that GIP infusion elicits a specific change of the activated gene set (ESM Table 3, ESM Fig. 2). When performing a PCA to group genes, five components were extracted. In a highly significant manner, each experimental setup was strongly correlated with only one of these components (ESM Table 3).

The comparison of saline and GIP infusion experiments showed the exchange of genes between specific modules involved, e.g. in GIPR downstream signalling pathways like G-protein coupled receptor signalling, cAMP signalling and extracellular related kinase (ERK) activation, pointing to activation of these genes or rearrangement of their functional context. Furthermore, cytokines like MCP-1 and their downstream pathways were involved, providing evidence for a GIP-dependent modulation of the inflammatory state in human adipose tissue.

Iterative group analysis With respect to the hypothesis that GIP might influence risk factors of the metabolic syndrome and adipogenesis via alteration of the inflammatory state in adipose tissue, we found several gene networks upregulated by GIP, which are linked to inflammatory markers as well as to chemokine and cytokine action. Details are shown in ESM Table 4.

These pathways contained members like $M C P-1, M C P-2$, $M I P-2, I L-1 \beta$ and $I L-6$ as well as their downstream pathways and thus may be important in the context of a GIP-dependent modulation of the inflammatory state in human adipose tissue.

Indeed, GIP infusion during high-insulin conditions increased $M C P-1$ and $M C P-2$ mRNA expression twofold and 2.6-fold, respectively, compared with saline ( $p<0.05$ after correction for multiple testing, Table 1). $M C P-1$ mRNAexpression was also twofold upregulated after GIP infusion during high-glucose/insulin conditions compared with saline treatment $(p<0.05)$. A similar tendency was observed for MCP-2 (Table 1). Neither insulin alone in EU clamps nor insulin and glucose in $\mathrm{HC}$ clamps caused significant increases of inflammatory cytokines.

Real-time qPCR of inflammatory marker expression To confirm the microarray data and to investigate the expression of specific inflammatory markers in more detail, we performed real-time qPCR of mRNA from hSCAT. $M C P-1$, $M C P-2$ and also $I L-6$ mRNA expression behaved very similarly with respect to GIP treatment and intervention conditions. A GIP infusion of 240 min duration was sufficient to increase the mRNA expression of $M C P-1 \quad(180 \pm 26 \%$, Fig. 1a), $M C P-2(246 \pm 58 \%$, ESM Fig. 3a) and $I L-6(234 \pm$ $40 \%$, Fig. 2a) significantly compared with baseline and also with saline infusion after $240 \min (p<0.05)$. Remarkably, prolonged elevation of plasma insulin or plasma insulin/ 
Table 1 Results of $M C P-1$ and $M C P-2$ gene expression in microarray analysis. Expression of regulated genes are presented as fold changes during clamps and infusions ( 240 vs $0 \mathrm{~min}$ ). The $p$ values are shown without and after Benjamini-Hochberg correction

\begin{tabular}{|c|c|c|c|c|c|c|}
\hline \multirow[t]{2}{*}{ Variable } & \multicolumn{3}{|l|}{$\mathrm{NaCl}$} & \multicolumn{3}{|l|}{ GIP } \\
\hline & Fold change & $p$ value & Corrected $p$ value & Fold change & $p$ value & Corrected $p$ value \\
\hline \multicolumn{7}{|l|}{ Infusion } \\
\hline$M C P-1$ & 1.34 & 0.268 & 0.418 & 1.54 & 0.128 & 0.273 \\
\hline$M C P-2$ & 1.57 & 0.152 & 0.338 & 2.29 & $0.031^{*}$ & 0.160 \\
\hline \multicolumn{7}{|l|}{ EU clamp } \\
\hline$M C P-1$ & 1.34 & 0.361 & 0.422 & 2.02 & $0.003 * *$ & $0.031^{*}$ \\
\hline$M C P-2$ & 1.66 & 0.186 & 0.300 & 2.66 & $<0.001 * * *$ & $0.013 *$ \\
\hline \multicolumn{7}{|l|}{ HC clamp } \\
\hline$M C P-1$ & 1.60 & 0.105 & 0.267 & 1.96 & $0.003 * *$ & $0.048 *$ \\
\hline$M C P-2$ & 1.53 & 0.117 & 0.278 & 1.71 & $0.020^{*}$ & 0.129 \\
\hline
\end{tabular}

${ }^{*} p<0.05,{ }^{*} p<0.01, * * * p<0.001$
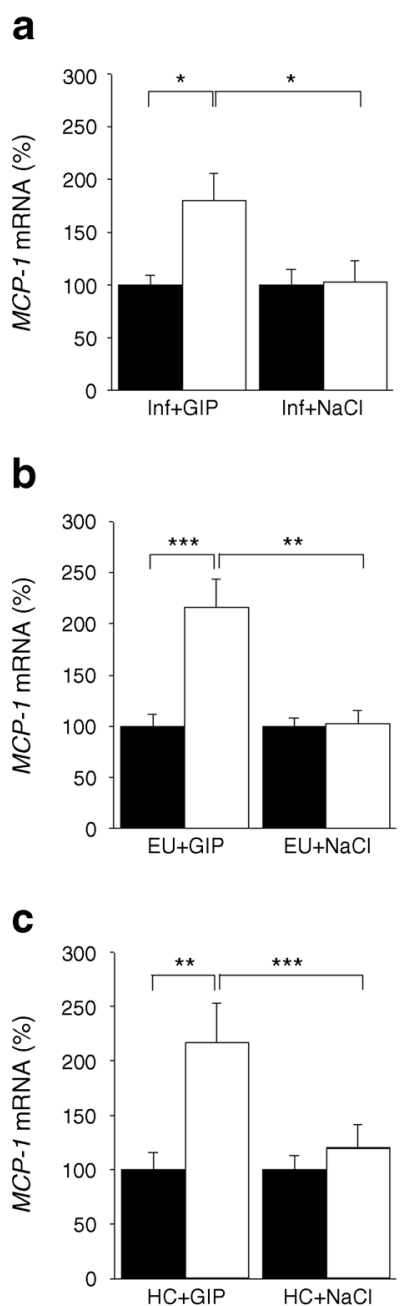

Fig. $1 M C P-1$ mRNA expression in human adipose tissue before and after GIP exposure for $240 \mathrm{~min}$ in comparison with saline $(\mathrm{NaCl})$ treatment. (a) Infusion (Inf) of GIP and saline only; (b) EU clamps; (c) HC clamps. Human plasma MCP-1 concentrations at 0, 120 and $240 \mathrm{~min}$ after infusion of saline or GIP: (d) infusion of GIP or saline only, (e) EU clamps, (f) $\mathrm{HC}$ clamps. Black bars, $0 \mathrm{~min}$; grey bars, $120 \mathrm{~min}$; white bars, 240 min. $* p<0.05,{ }^{* *} p<0.01 ; * * * p<0.001$
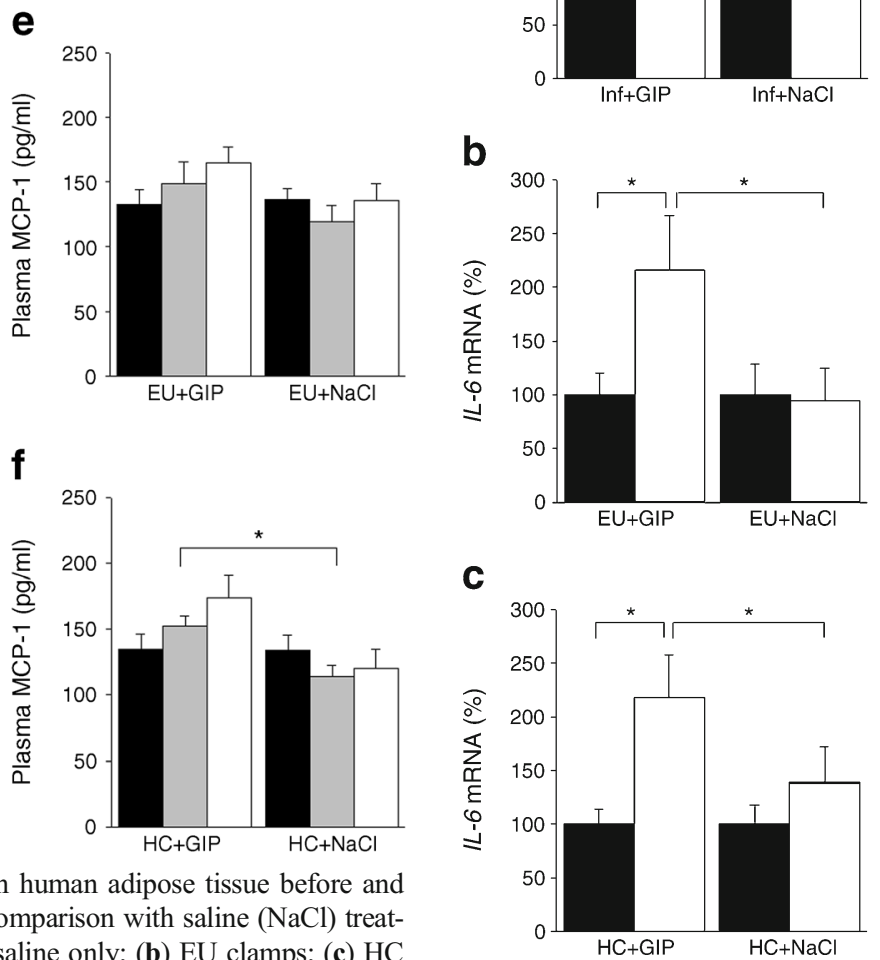

f a

d
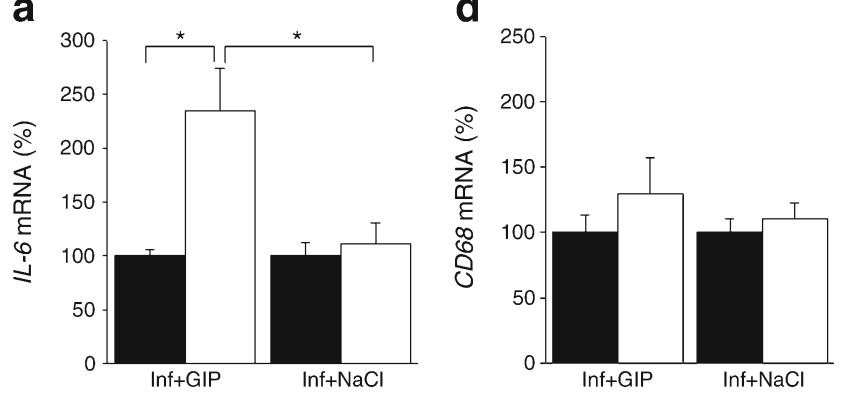

b

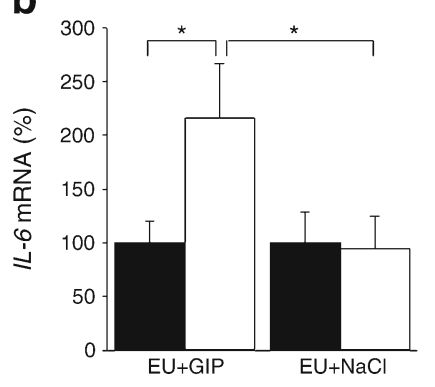

e

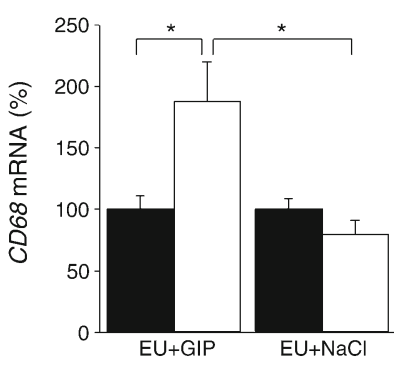

f

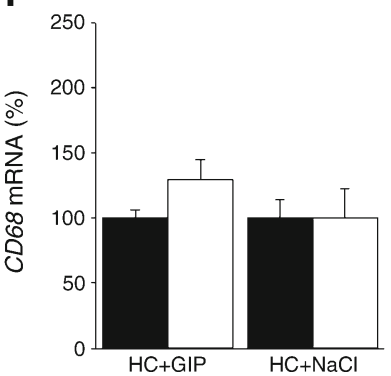

Fig. 2 IL-6 (a-c) and CD68 (d-f) mRNA expression in human adipose tissue before and after GIP exposure for $240 \mathrm{~min}$ in comparison to saline $(\mathrm{NaCl})$ treatment. (a, d) GIP and saline only; (b, e) EU clamps; (c, f) HC clamps. Black bars, 0 min; white bars, $240 \mathrm{~min} .{ }^{*} p<0.05$ 
glucose concentrations only marginally influenced the highly significant GIP-dependent elevation of $M C P-1, I L-6$ and $M C P-2$ mRNA expression (Figs 1, 2b,c and ESM Figs 3b, c) proven by ANOVA (adjusted for age, WHR, plasma glucose and insulin concentration) showing significant effects exclusively upon GIP administration (Table 2). The CD68 mRNA expression was significantly increased by GIP only under high-insulin conditions compared with baseline $(188 \pm 32 \%)$ and saline infusion ( $p<0.05$, respectively) (Fig. $2 \mathrm{~d}-\mathrm{f}$ ). In each intervention setup, saline infusion caused only minimal and insignificant changes in mRNA expression of $M C P-1, M C P-2, I L-6$ and $C D 68$. Interestingly, the $M C P-1$ receptor and GIPR were not regulated by GIPinfusion (data not shown).

\section{Human plasma MCP-1 concentrations under GIP} exposure GIP infusions were accompanied by significant increases of MCP-1 at $120 \mathrm{~min}(p<0.05)$ and $240 \mathrm{~min}(p<0.01)$ compared with baseline or compared with saline infusion after $240 \min (p<0.01$; Fig. 1d). GIP also resulted in increasing MCP-1 plasma concentrations after 120 or 240 min during high-glucose/insulin and high-insulin conditions compared with saline treatment, which was significant in the former (Fig. 1f; $120 \mathrm{~min}, p<0.05$ ) but not the latter case (Fig. 1e).

\section{Correlation of human plasma MCP-1 concentrations with} identified gene modules The difference in human plasma MCP-1 concentrations during $240 \mathrm{~min}$ of GIP infusion was correlated with the gene expression in the modules specified by WGCNA for each experimental setup. When comparing the sole GIP infusion with high-glucose/insulin and highinsulin conditions combined with GIP infusion, we identified a set of 13 genes, which changed into the gene module most highly correlated with plasma MCP-1 concentrations under each experimental setup (ESM Table 5). This set of 13 genes, regarded as mainly GIP driven genes independent from glucose and insulin concentration, was involved in processes of G-protein coupled receptor mediated transmission of cytokine receptor signalling ( $p=0.0157$; ESM Fig. 4).

\section{Effect of GIP on MCP-1 mRNA expression in human and murine adipocyte/macrophage co-cultures and media-} transfer experiments Stimulation of human THP-1 monocytes/macrophages, primary human macrophages, mouse RAW2 64.7 macrophages or human SGBS adipocytes and differentiated mouse 3T3-L1 adipocytes with $100 \mathrm{nmol} / 1$ GIP for up to 240 min did not lead to any significant increase of $M C P-1$ mRNA. Since a mutual induction of $M c p-1$ mRNA was reported in 3T3-L1/RAW 264.7 co-cultures [6], we tested whether GIP is able to enhance $M C P-1$ mRNA production under these conditions in murine and human cells. Indeed, in 3T3-L1/RAW 264.7 as well as in human SGBS/primary macrophage co-cultures, GIP significantly increased $M C P-1$
mRNA expression after 240 min compared with saline ( $p<0.05$; Fig. 3).

Crossover media-transfer experiments from 3T3-L1 and RAW 264.7 cells, to determine the prominent partner and direction of signalling, revealed that the transfer of media from macrophages pre-stimulated with $100 \mathrm{nmol} / \mathrm{l} \mathrm{GIP}$ for $240 \mathrm{~min}$, to adipocytes, led to a much higher expression of $M c p-1$ after an additional $240 \min (134 \pm 42$-fold; $p=0.035)$, than vice versa transfer ( $1.7 \pm 0.3$-fold; $p=0.134$; Fig. $3 \mathrm{~d})$. With separate incubation of 3T3-L1 or RAW 264.7 cells with either $0.9 \%$ $\mathrm{NaCl}$ or $100 \mathrm{nmol} / 1$ GIP, Mcp-1 gene expression increased only $1.3 \pm 0.2$-fold in $3 \mathrm{~T} 3$-L1 and $2.1 \pm 0.4$-fold in RAW 264.7 ( $p<0.05$ for RAW 264.7; Fig. 3c).

Furthermore, on a cytokine array, Mip-1 $\beta$ was the only member with a significantly higher concentration in the supernatant fraction of RAW 264.7 (1.43 \pm 0.03 -fold) prestimulated with $100 \mathrm{nmol} / \mathrm{l}$ GIP for $240 \mathrm{~min}$, but not in the supernatant fraction of 3T3-L1 cells $(1.13 \pm 0.03$-fold; $p<0.01$ RAW 264.7 vs 3T3-L1).

GIPR mRNA and protein expression in human monocytes and murine macrophages Besides human and mouse a

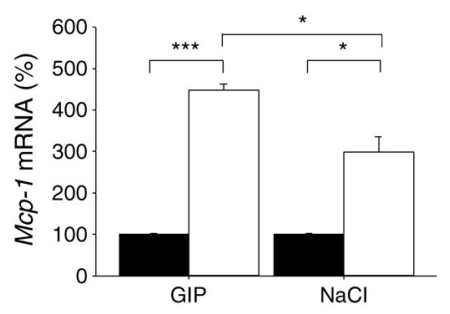

C

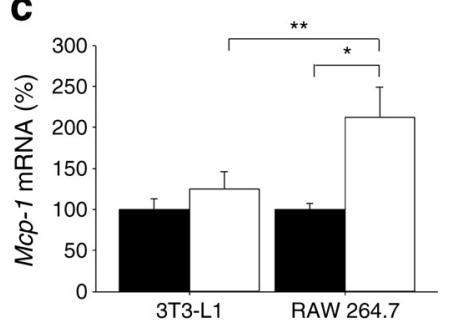

b

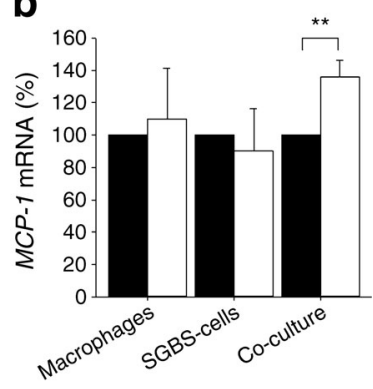

d

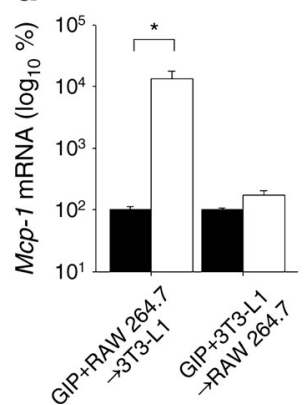

Fig. $3 M C P-1$ mRNA expression with saline $(\mathrm{NaCl})$ or stimulated with GIP $(100 \mathrm{nmol} / \mathrm{l})$ for $240 \mathrm{~min}$ in (a) co-culture of 3T3-L1 adipocytes and RAW 264.7 cells $(n=3$; GIP + diprotin A; black bars, 0 min; white bars, $240 \mathrm{~min}$ ); (b) human SGBS cells and primary macrophages separately and in co-culture ( $n=5$; black bars, $\mathrm{NaCl}$; white bars, D2Ala-GIP); and (c) RAW 264.7 cells and 3T3-L1 ( $n=3$; black bars, $\mathrm{NaCl}$; white bars, GIP + diprotin A). (d) Transfer of media-supernatant fraction from GIP pre-treated (240 $\mathrm{min}$ ) RAW 264.7 cells to 3T3-L1 cells for additional $240 \mathrm{~min}$ or transfer of media-supernatant fraction from GIP pre-treated (240 min) 3T3-L1 cells to RAW 264.7 cells for additional $240 \mathrm{~min}(n=3$ independent experiments; GIP + diprotin A; black bars, 0 min; white bars, $240 \mathrm{~min}) .{ }^{*} p<0.05, * * p<0.01, * * * p<0.001$ 
adipocytes, GIPR gene expression was recently shown in human monocytes or macrophages [28]. We additionally detected GIPR mRNA expression in human THP-1 macrophage (Fig. 4a) cells and murine RAW macrophages (data not shown), and GIPR protein in human THP-1 monocytes, differentiated THP-1 macrophages, human adipose tissue samples, human primary blood monocytes and in murine RAW 264.7 cells (Fig. 4b). However, GIPR protein was not detectable in human primary monocyte-derived macrophages matching the also much lower mRNA levels.

\section{Analysis of GIPR function in monocytes and} macrophages Since GIP stimulation of insulin secretion from pancreatic beta cells involves activation of adenylate cyclase and the p44/42 ERKs [29], we measured the cAMP concentration and ERK phosphorylation in human primary blood monocytes, THP-1 and RAW 264.7 cells after stimulation with GIP, using INS-1 cells as positive controls.

GIP stimulation indicated functional GIPR by increasing the cAMP concentration in primary human blood monocytes $(p<0.05)$, THP-1 monocytes $(p<0.05)$ and RAW 264.7 macrophages $(p<0.001)$ significantly (Fig. 5a). Furthermore, incubation with D2Ala-GIP did not alter p42/p44-ERK phosphorylation in human primary monocytes and macrophages (Fig. 5b) but GIP + diprotin A led to a highly significant increase in the ratio of phosphorylated to total p42/p44-ERK in the THP1 and RAW 264.7 macrophages as well as in INS-1 cells (Fig. 5c-e).

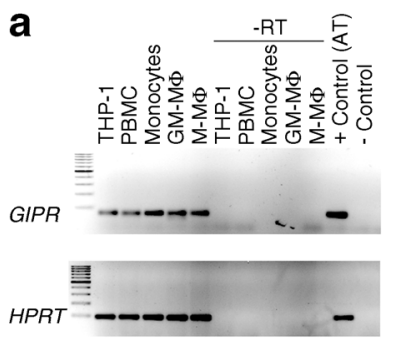

b

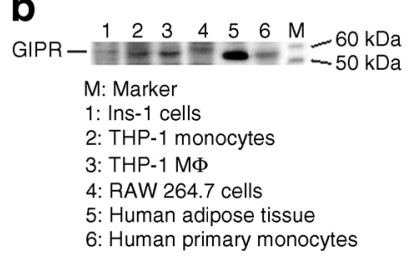

Fig. 4 (a) GIPR mRNA expression normalised to hypoxanthine-guanine phosphoribosyltransferase (HPRT) in THP-1 macrophages (M $\Phi)$ and human PBMC, monocytes and monocyte-derived macrophages from the same donors $(n=3)$; (b) GIPR protein levels in lysates of INS-1, THP-1 monocytes, THP-1 macrophages, RAW 264.7 cells, human adipose tissue and human monocytes detected by western blotting. In all lysates the signal was reduced by pre-incubation of the GIPR antibody with the specific blocking peptide (data not shown). AT, adipose tissue; GM$\mathrm{M} \Phi$, granulocyte macrophage colony-stimulating factor derived; M$\mathrm{M} \Phi$, macrophage colony-stimulating factor derived

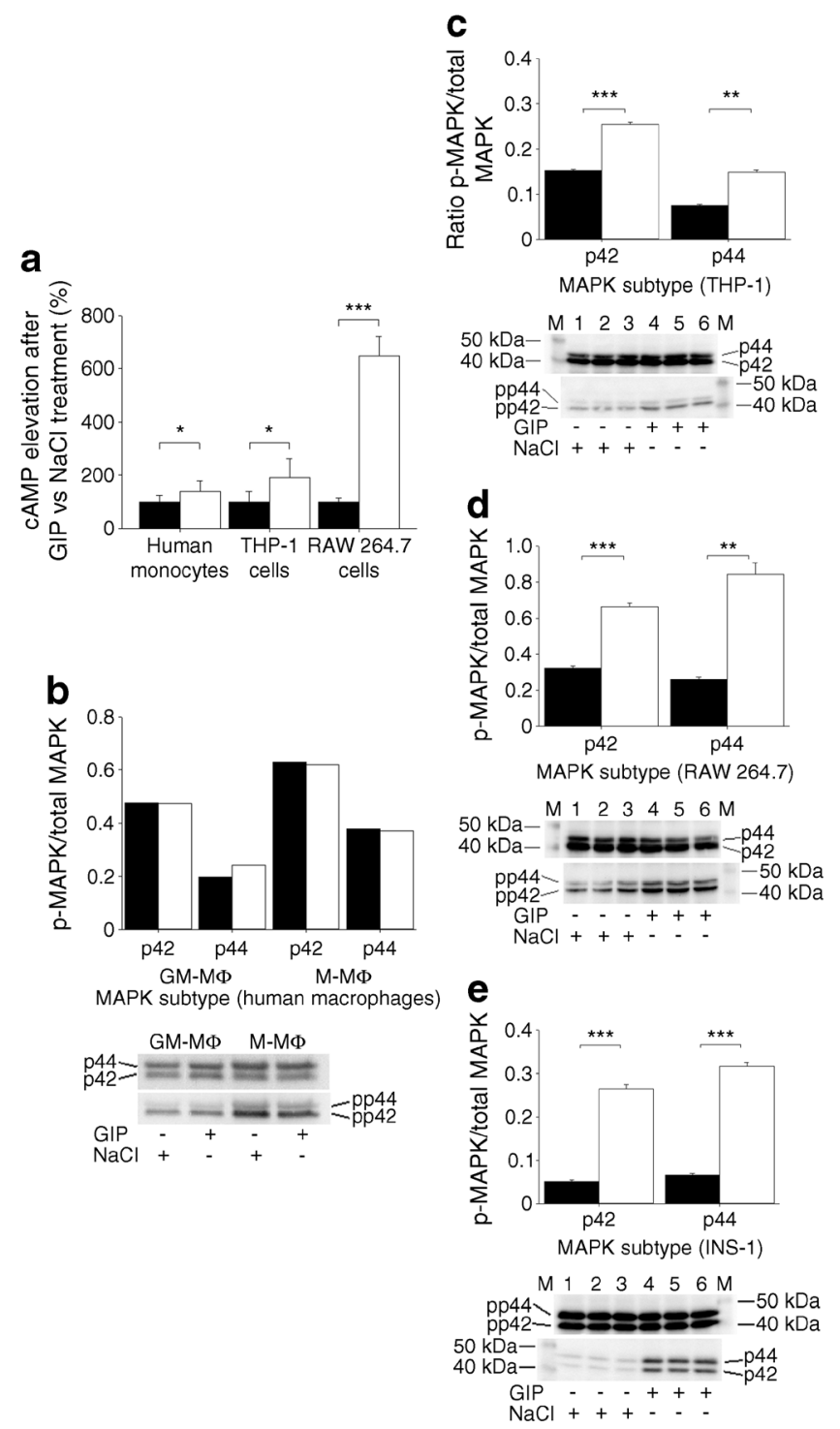

Fig. 5 (a) Maximum stimulation (white bars) of cAMP production compared with baseline (black bars) in human primary blood monocytes, THP-1 monocytes and RAW 264.7 macrophages after incubation with GIP + diprotin A (100 nmol/1 GIP; $n=3$; values from saline $[\mathrm{NaCl}+$ diprotin $\mathrm{A}]$ stimulation were subtracted; $p<0.05$ ). The cells were incubated for $30 \mathrm{~min}$. The maximum stimulation was reached after 10-15 min. (b-e) Western blot and densitometric representation of phosphorylated and total p42-ERK2 and p44-ERK1 in protein lysates of (b) human monocyte-derived macrophages, (c) THP-1 macrophages, (d) RAW 264.7 macrophages and (e) INS-1 cells after 30 min incubation with either (c-e) $0 \mathrm{nmol} / \mathrm{l}(\mathrm{NaCl}$, black bars) or $100 \mathrm{nmol} / \mathrm{l} \mathrm{GIP}$ with diprotin $\mathrm{A}$ (white bars) or (b) $100 \mathrm{nmol} / 1$ D2Ala-GIP. Representative blots are shown $(n=3) . * p<0.05, * * p<0.01, * * * p<0.001$. GM-M $\Phi$, granulocyte macrophage colony-stimulating factor derived; $\mathrm{M}-\mathrm{M} \Phi$, macrophage colonystimulating factor derived

\section{Discussion}

The present study demonstrates for the first time that GIP rapidly induces a broad inflammatory response involving the chemokines MCP-1 and MCP-2 as well as IL-6 in human 
adipose tissue in vivo. Furthermore, we show that functional GIPRs are expressed by primary human monocytes and both human and murine monocyte/macrophage cell lines in addition to the established expression in human and murine adipocytes, and suggest a model for the GIP-induced initiation of inflammatory response in adipose tissue.

Inflammatory signals originating from adipose tissue macrophages are accepted components in the development of insulin resistance and its consequent diseases such as type 2 diabetes and atherosclerosis [30]. In this context, overnutrition is primarily viewed as a cause of obesity, which then attracts macrophages in relation to the mass of adipose tissue [2, 31]. MCP-1 and MCP-2 were assigned an important role in both attracting macrophages to adipose tissue [11] and in determining a more inflammatory M1 macrophage phenotype. However, the initiation of macrophage recruitment into adipose tissue remained largely unclear [32].

Investigation of regulated gene networks from microarray data revealed several GIP-driven clusters of inflammatory genes containing cytokines, their receptors and downstream signalling components in hSCAT. A set of 13 glucose- and insulin-independent but GIP-driven genes was highly correlated with the change of human plasma MCP-1 concentrations during all experimental setups. In addition, microarray and real-time qPCR analysis of specific inflammatory markers like $M C P-1, M C P-2, I L-6$ and $C D 68$ in hSCAT biopsies uncovered putative mediators of a GIP effect on inflammation (Table 2).

The ANOVA model and real-time qPCR showed that the significant and rapid increase in mRNA expression of $M C P-1$, $M C P-2$ and $I L-6$ in hSCAT was not evident in response to elevated insulin or elevated glucose or both, indicating that the hormonal GIP stimulus rather than the metabolic stimulus was causal in our setting. Remarkably, the increase in $M C P-1$ mRNA in adipose tissue was translated into increased circulating plasma levels of MCP-1 in humans suggesting systemic relevance of this stimulus.

Furthermore, we found an upregulation of $I L-6$ and of the macrophage marker $C D 68$ in adipose tissue indicating a promotion of macrophage recruitment by GIP. MCP-1 and MCP2 are produced by macrophages as well as by adipocytes.
MCP-1 and its receptor, $\mathrm{C}-\mathrm{C}$ chemokine receptor (CCR) type 2 (CCR2), are highly expressed in adipose tissue [32]. Overexpression of $M c p-1$ in mouse adipose tissue increased circulating levels of MCP-1 and induced macrophage accumulation $[9,10]$. Our data, especially from cell culture experiments in murine 3T3-L1 and RAW 264.7 macrophages, raised the question of whether macrophages may be a potential target of GIP. We were able to confirm GIPR expression on mRNA and protein level as well as their functionality by measurement of cAMP concentration and phosphorylation of p42/p44 MAPKs [29] in human primary blood monocytes, the human monocyte/macrophage cell line THP-1, murine macrophage cell line RAW 264.7 and the GIPR-expressing insulinoma cell line INS-1 (as a control).

Similar to a study where Timper et al demonstrated increased gene expression of $I L-1 \beta, I L-6$ and $I L-1 R A$ but not $M C P-1$ in response to GIP in cultured human primary adipocytes [25], we did not see a significant increase of MCP-1 expression in our THP-1 and RAW 264.7 cells, human macrophages, differentiated 3T3-L1 or human SGBS cells in response to GIP exposure. The obvious difference in GIP-dependent in vivo $M C P-1$ expression between human adipose tissue biopsies and culture of single cell lines led us to the reasonable suspicion that a crosstalk between adipocytes and resident macrophages is necessary for a physiological inflammatory response to GIP. Indeed, our human and murine adipocyte/ macrophage co-culture systems to mimic the in vivo situation showed an increase in $M C P-1$ mRNA expression in response to GIP treatment. The fact that only the mediasupernatant fraction from GIP-stimulated macrophages was able to induce a solid $M c p-1$ expression after transfer to adipocytes but not vice versa, suggests a secreted soluble factor to finally initiate the crosstalk from macrophages to adipocytes. The more potent effect of media transfer on Mcp-1 expression than in co-culture can be explained by a macrophage/adipocyte ratio of 50:50 in media-transfer experiments compared with 10:50 in co-cultures. Two results make macrophage inflammatory protein- $1 \beta$ (MIP$1 \beta)$ a potential candidate to be at least one of the signal transmitters from macrophages to adipocytes: the significant upregulation of MIP-1 $\beta$ protein expression after GIP

Table 2 Real-time $\mathrm{qPCR}$ data ${ }^{\mathrm{a}}$ and MCP-1 plasma concentrations

\begin{tabular}{lllllll}
\hline Variable & \multicolumn{2}{l}{ Gene expression (real-time qPCR) } & & \multicolumn{2}{c}{ Plasma concentration } \\
\cline { 2 - 6 } & $M C P-1$ & $M C P-2$ & $I L-6$ & $C D 68$ & $M C P-1$ receptor & MCP-1 \\
\hline Setup (infusion vs EC vs HC) & 0.265 & 0.788 & 0.640 & 0.670 & 0.260 & 0.815 \\
Treatment (GIP vs NaCl) & $0.008^{* *}$ & $0.014^{*}$ & $0.023^{*}$ & $0.038^{*}$ & 0.882 & $0.035^{*}$ \\
\hline
\end{tabular}

${ }^{\mathrm{a}}$ ANOVA, adjusted for age, WHR, plasma glucose and insulin concentration

${ }^{*} p<0.05,{ }^{* *} p<0.01$ 


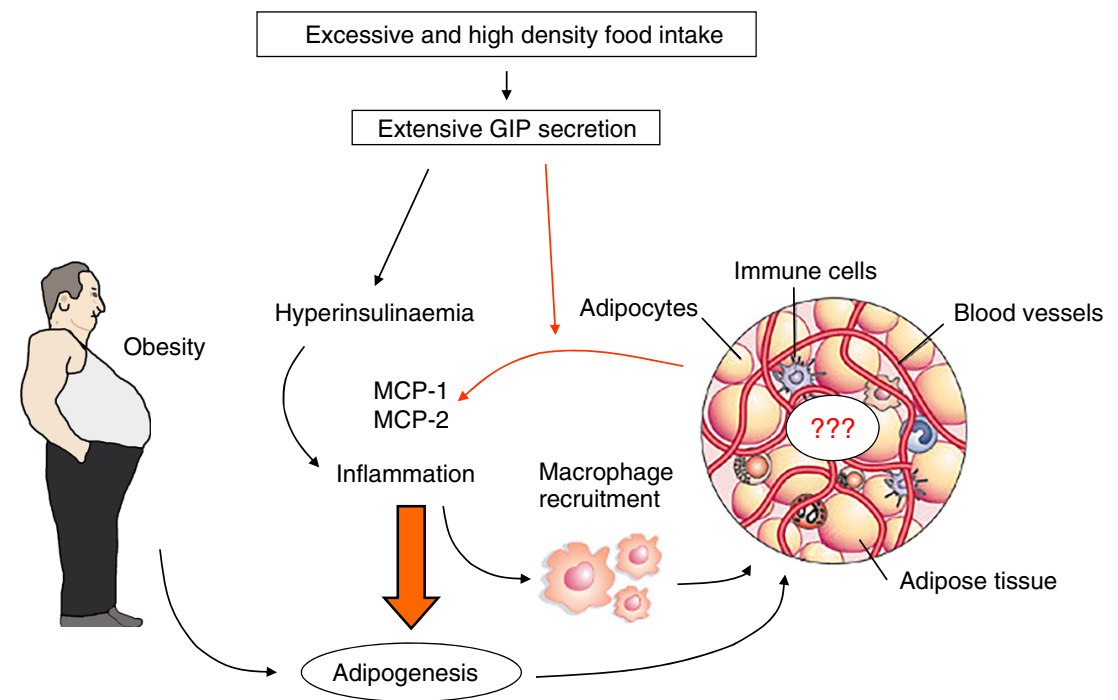

Fig. 6 Postprandial release of GIP induces adipose tissue inflammation and contributes to the proinflammatory and 'unhealthy' character of excessive food intake. Postprandial insulin and GIP plasma levels are physiologically elevated. These levels decline rapidly after moderate meals, while large and energy dense meals induce pronounced and prolonged release of GIP. GIP may then induce the synthesis and release of MCP-1/ 2 , which promotes macrophage recruitment and inflammation in adipose tissue thereby contributing to the 'unhealthy character' of the meal stimulation of RAW 264.7 but not 3T3-L1 cells, and the involvement of the previously mentioned set of 13 GIP-driven genes (ESM Fig. 4) linked to human plasma MCP-1 concentrations in the signal transduction of the MIP- $1 \beta$ receptor (CCR4).

GIPR antagonism or genetic deletion prevents weight gain and metabolic abnormalities associated with high fat or energy dense diet induced obesity in mice $[16,33]$ while these diets are also powerful stimulants of $M c p-1$ in mice in vivo [34]. In $\mathrm{Ob} / \mathrm{Ob}$ mice, deletion of Gipr or treatment with GIP antagonists leads to major improvements of metabolism and insulin sensitivity despite persisting obesity $[16,17,35]$. In humans, surgical bypass of GIP-secreting K-cells greatly decreases GIP levels and results in rapid improvement of insulin sensitivity [35-37].

The direct GIP-induced elevation of $M C P-1$ mRNA expression as well as circulating MCP-1 plasma concentrations we found in this study may be the link between chronically or frequently elevated GIP concentrations by ingestion of energy dense food or several intermediate snacks and inflammatory responses and insulin resistance.

In summary, we here demonstrated for the first time functional GIPR expression on monocytes and macrophages and introduce in vivo the concept that periprandially released hormones, particularly GIP, play a crucial role in initiating low level inflammation associated with obesity. GIP appears to promote a crosstalk between macrophages and adipocytes to induce this response. These observations suggest that food inducing extensive release of GIP may be proinflammatory due to the hormonal response it induces and imply novel approaches to conceive healthy foods with a low GIP releasing potential (Fig. 6). GIPR and MCP-1 receptor antagonists might prove beneficial to reduce inflammation linked with obesity, and in prevention of type 2 diabetes and the metabolic syndrome.

Acknowledgements We wish to thank A. Wagner, A. Borchert and K. Sprengel in our department for technical assistance. We also thank S. Sucher in our department for her excellent technical support. RAW 264.7 cells were kindly provided by A. Kiderlen (Robert-Koch Institute, Berlin, Germany). SGBS cells were kindly provided by M. Wabitsch (University Medical Center, Ulm, Germany). D2Ala-GIP was a gift from V. Gault (School of Biomedical Sciences, University of Ulster, Coleraine campus, Coleraine, UK).

Funding This work was supported by the German Research Foundation (DFG): grant No. Pf164/021002. The funders had no role in the study design, data collection and analysis, the decision to publish, or preparation of the manuscript.

Duality of interest The authors declare that there is no duality of interest associated with this manuscript.

Contribution statement ÖG, NR, MN and AFHP contributed to the conception and design, AFHP to the supervision of the study. ÖG contributed to the clinical study, acquisition of adipose tissue biopsies and human blood samples. ÖG and MAO contributed to the investigation of human samples from the clinical study and cell culture experiments. ÖG, MAO, ACS, OP and RS contributed to cellular co-culture experiments. ASM contributed to microarray hybridisation and acquisition of microarray data. ÖG and MAO contributed to the evaluation and statistical analysis of microarray data. OP, MK and ACS contributed to the investigation of human primary monocytes and macrophages. ÖG, MAO, ASM, RS and MK contributed to the analysis of the data. ÖG, MAO, OP and AFHP contributed to the drafting. All authors contributed to the critical review of the manuscript and approved the final version. ÖG and MAO are the guarantors of this work. 


\section{References}

1. Xu H, Barnes GT, Yang Q et al (2003) Chronic inflammation in fat plays a crucial role in the development of obesity-related insulin resistance. J Clin Invest 112:1821-1830

2. Weisberg SP, McCann D, Desai M, Rosenbaum M, Leibel RL, Ferrante AW Jr (2003) Obesity is associated with macrophage accumulation in adipose tissue. J Clin Invest 112:1796-1808

3. Hotamisligil GS, Budavari A, Murray D, Spiegelman BM (1994) Reduced tyrosine kinase activity of the insulin receptor in obesitydiabetes. Central role of tumor necrosis factor-alpha. J Clin Invest 94:1543-1549

4. Uysal KT, Wiesbrock SM, Marino MW, Hotamisligil GS (1997) Protection from obesity-induced insulin resistance in mice lacking TNF-alpha function. Nature 389:610-614

5. Sartipy P, Loskutoff DJ (2003) Monocyte chemoattractant protein 1 in obesity and insulin resistance. Proc Natl Acad Sci U S A 100: 7265-7270

6. Suganami T, Nishida J, Ogawa Y (2005) A paracrine loop between adipocytes and macrophages aggravates inflammatory changes: role of free fatty acids and tumor necrosis factor alpha. Arterioscler Thromb Vasc Biol 25:2062-2068

7. Odegaard JI, Ricardo-Gonzalez RR, Goforth MH et al (2007) Macrophage-specific PPARgamma controls alternative activation and improves insulin resistance. Nature 447:1116-1120

8. Arkan MC, Hevener AL, Greten FR et al (2005) IKK-beta links inflammation to obesity-induced insulin resistance. Nat Med 11: 191-198

9. Kamei N, Tobe K, Suzuki R et al (2006) Overexpression of monocyte chemoattractant protein-1 in adipose tissues causes macrophage recruitment and insulin resistance. J Biol Chem 281: 26602-26614

10. Kanda H, Tateya S, Tamori Y et al (2006) MCP-1 contributes to macrophage infiltration into adipose tissue, insulin resistance, and hepatic steatosis in obesity. J Clin Invest 116:1494-1505

11. Weisberg SP, Hunter D, Huber R et al (2006) CCR2 modulates inflammatory and metabolic effects of high-fat feeding. J Clin Invest 116:115-124

12. Elliott RM, Morgan LM, Tredger JA, Deacon S, Wright J, Marks V (1993) Glucagon-like peptide-1 (7-36)amide and glucosedependent insulinotropic polypeptide secretion in response to nutrient ingestion in man: acute post-prandial and 24-h secretion patterns. J Endocrinol 138:159-166

13. Jones IR, Owens DR, Luzio SD, Hayes TM (1989) Obesity is associated with increased post-prandial GIP levels which are not reduced by dietary restriction and weight loss. Diabete Metab 15: $11-22$

14. Baggio LL, Drucker DJ (2007) Biology of incretins: GLP-1 and GIP. Gastroenterology 132:2131-2157

15. Holst JJ, Vilsbøll T, Deacon CF (2008) The incretin system and its role in type 2 diabetes mellitus. Mol Cell Endocrinol 297:127-136

16. Miyawaki K, Yamada Y, Ban N et al (2002) Inhibition of gastric inhibitory polypeptide signaling prevents obesity. Nat Med 8: 738-742

17. Flatt PR (2008) Dorothy Hodgkin Lecture 2008. Gastric inhibitory polypeptide (GIP) revisited: a new therapeutic target for obesitydiabetes? Diabet Med 25:759-764

18. Hansotia T, Maida A, Flock G et al (2007) Extrapancreatic incretin receptors modulate glucose homeostasis, body weight, and energy expenditure. J Clin Invest 117:143-152

19. Mazzaferri EL, Starich GH, Lardinois CK, Bowen GD (1985) Gastric inhibitory polypeptide responses to nutrients in
Caucasians and American Indians with obesity and noninsulindependent diabetes mellitus. J Clin Endocrinol Metab 61:313-321

20. Hauner H, Glatting G, Kaminska D, Pfeiffer EF (1988) Effects of gastric inhibitory polypeptide on glucose and lipid metabolism of isolated rat adipocytes. Ann Nutr Metab 32:282-288

21. Kim SJ, Nian C, McIntosh CH (2007) Activation of lipoprotein lipase by glucose-dependent insulinotropic polypeptide in adipocytes. A role for a protein kinase B, LKB1, and AMP-activated protein kinase cascade. J Biol Chem 282:8557-8567

22. Song DH, Getty-Kaushik L, Tseng E, Simon J, Corkey BE, Wolfe MM (2007) Glucose-dependent insulinotropic polypeptide enhances adipocyte development and glucose uptake in part through Akt activation. Gastroenterology 133:1796-1805

23. Vilsbøll T, Holst JJ (2004) Incretins, insulin secretion and type 2 diabetes mellitus. Diabetologia 47:357-366

24. Rudovich N, Kaiser S, Engeli S et al (2007) GIP receptor mRNA expression in different fat tissue depots in postmenopausal nondiabetic women. Regul Pept 142:138-145

25. Timper K, Grisouard J, Sauter NS et al (2013) Glucosedependent insulinotropic polypeptide induces cytokine expression, lipolysis, and insulin resistance in human adipocytes. Am J Physiol Endocrinol Metab 304:E1-E13

26. Breitling R, Amtmann A, Herzyk P (2004) Iterative Group Analysis (iGA): a simple tool to enhance sensitivity and facilitate interpretation of microarray experiments. BMC Bioinforma 5:34

27. Zhang B, Horvath $\mathrm{S}$ (2005) A general framework for weighted gene co-expression network analysis. Stat Appl Genet Mol Biol 4: Article17

28. Pivovarova O, Hornemann S, Weimer S et al (2015) Regulation of nutrition-associated receptors in blood monocytes of normal weight and obese humans. Peptides 65C:12-19

29. Ehses JA, Pelech SL, Pederson RA, McIntosh CH (2002) Glucosedependent insulinotropic polypeptide activates the Raf-Mek1/2ERK1/2 module via a cyclic AMP/cAMP-dependent protein kinase/Rap1-mediated pathway. J Biol Chem 277:37088-37097

30. Fernandez-Real JM, Pickup JC (2008) Innate immunity, insulin resistance and type 2 diabetes. Trends Endocrinol Metab 19:10-16

31. Wellen KE, Hotamisligil GS (2005) Inflammation, stress, and diabetes. J Clin Invest 115:1111-1119

32. Neels JG, Olefsky JM (2006) Inflamed fat: what starts the fire? J Clin Invest 116:33-35

33. McClean PL, Irwin N, Cassidy RS, Holst JJ, Gault VA, Flatt PR (2007) GIP receptor antagonism reverses obesity, insulin resistance, and associated metabolic disturbances induced in mice by prolonged consumption of high-fat diet. Am J Physiol Endocrinol Metab 293:E1746-E1755

34. Jiao P, Chen Q, Shah S et al (2009) Obesity-related upregulation of monocyte chemotactic factors in adipocytes: involvement of nuclear factor-kappaB and c-Jun NH2-terminal kinase pathways. Diabetes 58:104-115

35. Gault VA, Irwin N, Green BD et al (2005) Chemical ablation of gastric inhibitory polypeptide receptor action by daily (Pro3)GIP administration improves glucose tolerance and ameliorates insulin resistance and abnormalities of islet structure in obesity-related diabetes. Diabetes 54:2436-2446

36. Korner J, Bessler M, Inabnet W, Taveras C, Holst JJ (2007) Exaggerated glucagon-like peptide- 1 and blunted glucosedependent insulinotropic peptide secretion are associated with Roux-en-Y gastric bypass but not adjustable gastric banding. Surg Obes Relat Dis 3:597-601

37. Mingrone G, Nolfe G, Gissey GC et al (2009) Circadian rhythms of GIP and GLP1 in glucose-tolerant and in type 2 diabetic patients after biliopancreatic diversion. Diabetologia 52:873-881 